\title{
Comorbidity indices: a call for the integration of physical and mental health
}

\author{
Molly A. Nowels ${ }^{1}$ and Lynn M. VanderWielen ${ }^{2}$ \\ ${ }^{1}$ Department of Family Medicine, Colorado School of Public Health, University of Colorado School of Medicine, Aurora, \\ CO, USA \\ ${ }^{2}$ Department of Family Medicine, Mental Health Center of Denver, University of Colorado School of Medicine, Aurora, \\ CO, USA
}

\begin{abstract}
Comorbidity indices are commonly used in health services research as a measure of, or as a control for, the severity of a person's medical state. Currently, there is not a comorbidity index for mental health diagnoses, despite the fact that almost half of Americans have a diagnosable mental health condition at least once in their lifetime. This commentary calls for the integration of mental and behavioral health in comorbidity indices to appropriately account for the role of mental health in overall morbidity and mortality.
\end{abstract}

Key words: administrative data; comorbidity; mental health; physical health

Received 22 September 2016; revised 15 May 2017; accepted 17 June 2017; first published online 20 July 2017

Administrative data sets, including Electronic Medical Records (EHR) extractions and insurance claim databases, are commonly used in the health services research (HSR) literature to examine clinical, population health, and programmatic research questions. One commonly employed method to control for patient disease severity is to include a comorbidity or severity index. Two such methods, the Charlson Comorbidity Index (Charlson et al., 1987) and the Elixhauser Comorbidity Measure (Elixhauser et al., 1998) have been jointly cited over 20000 times in the literature. Both measures provide a baseline medical comorbidity adjustment to researchers and estimates of clinical prognosis to clinicians. In this paper, the term comorbidity will be used. However, a more apt term may be 'multimorbidity,' as this does not connote an index disease or any type of causal relationship between diseases, as comorbidity can.

The Charlson Comorbidity Index was created to prospectively evaluate the impact of multiple conditions on the risk of mortality for longitudinal

Correspondence to: Molly A. Nowels, Department of Family Medicine, University of Colorado School of Medicine, Mail stop F496, AO1, 12631 E. 17th Ave., Aurora, CO 80045, USA. Email: molly.nowels@ucdenver.edu studies (Charlson et al., 1987). In addition to predicting risk of death, the Charlson has been used to predict outcomes such as complications, length of stay during inpatient visit, and financial metrics (Klabunde et al., 2000). Validated as an approach to risk adjustment in claims-based research using International Classification of Disease (ICD)-9 codes (D'Hoore et al., 1996), the Charlson Comorbidity Index is a useful tool in multiple areas of HSR and clinical prognosis. Calculated summary measures such as the Charlson Comorbidity Index assign weights to various medical conditions and then sum the weights present for each individual (Charlson et al., 1987). The sum of the weights represents the comorbidity score for each individual, with higher scores indicating higher 10 -year mortality risk and higher predicted resource use. Although originally designed to predict morbidity, such indices are currently more frequently used in research settings. Despite the many strengths of the Charlson Comorbidity Index and Elixhauser Comorbidty Measure, such measures impose significant challenges to researchers who are interested in, and cognizant to, behavioral health comorbidities.

Mental health and substance abuse conditions are pervasive in the United States and worldwide. For instance, the observed lifetime prevalence of

\footnotetext{
(C) Cambridge University Press 2017
} 
any mental health condition in the United States is $46.4 \%$ (Kessler et al., 2005a), while the annual prevalence of a diagnosable mental health condition in 2013 was $18.5 \%$ among US adults (SAMHSA, 2014). In fact, of those with at least one mental health disorder, almost half have two or more mental health disorders (Kessler et al., 2005b). It has been demonstrated that for those with a non-substance abuse mental health diagnosis, the odds of having a substance abuse disorder were 2.7 times higher than those without another mental health diagnosis, and among people with an alcohol addiction, $37 \%$ had a comorbid mental health diagnosis (Regier et al., 1990). In fact, individuals with mental health conditions incur significant increases in morbidity and mortality, such that the life expectancy of many groups of individuals with mental illnesses in high-income countries is at least $20 \%$ less than for the general population(Newman and Bland, 1991).

Not only are mental health and substance abuse comorbidities common occurrences but individuals with mental health conditions frequently sustain physical health comorbidities. For instance, among Medicaid recipients with serious and persistent mental illness, nearly $75 \%$ were diagnosed with at least one chronic health condition, and $50 \%$ were diagnosed with two or more chronic health conditions (Jones et al., 2004). Adults with severe mental illnesses are more likely to have several physical health conditions such as hypertension, diabetes, and lung diseases than their non-mental disordered counterparts (Sokal et al., 2004).The Robert Wood Johnson Foundation estimates that $68 \%$ of adults with a mental disorder have at least one medical condition, and $29 \%$ of adults with a medical condition have a mental disorder (Druss and Walker, 2011). Due to a myriad of factors including low detection rates and inequitable access to care, individuals with serious and persistent mental illness have higher morbidity and mortality rates than matched controls (Dickey et al., 2002; Boardman, 2006).

In addition to what the data from many studies has demonstrated, medicine as a field may be experiencing a philosophical shift away from treating the mind and body as separate entities.

As more diseases are found to have social and psychological underpinnings, medicine must adapt to treat individuals as a whole person, rather than a body separate from a mind (Mehta, 2011).
Several structural barriers hinder the integration of mental health measures into comorbidity indices, including documentation, and data extraction. Mental health professionals rely on the Diagnostic and Statistical Manual of Mental Disorders (DSM), currently in its fifth edition, whereas physical health providers lean on coding from the ICD, currently in its 10th edition. As both mental health and physical health providers may produce mental health diagnoses, a statistical model including a mental health severity measure would need to allow for the inclusion of either DSM-V or ICD-10 diagnoses. Additional consideration would be required to provide the appropriate variable weighting based on DSM-V or ICD-10 diagnoses, as the coding schemes to not directly correlate for disease severity or etiology.

The source of administrative data for research purposes is important to the considerations of mental health severity. Insurance data should include mental health diagnoses, and the Mental Health Parity Addiction and Equity Act requires comparable coverage for mental health concerns as physical health concerns, but data may not be characterized in the same way. Just as the coding scheme varies between the DSM-V and ICD-10, the data extraction team may need to pull additional variables besides traditionally defined diagnoses to ensure mental health diagnoses are properly captured in the data set. Electronic Medical Record data provides a much more challenging environment for ensuring the data extraction includes all variables of interest. Often, EHRs designed for physical health providers do not include fields traditionally used in the mental health space. Often the mental health provider may end up using text boxes to fill in treatment plans and referral diagnoses, rather than in standardized formats, making data extraction and data set creation difficult. Ideally, mental health providers and physical health providers will utilize the same software and software version for billing and coding, but this is traditionally the case among networked facilities. If a study team wants to follow patients throughout a geographic area, they will likely find that they will need to request a data extraction from several different EHR systems, which will lead to the challenge of aggregating one data set.

These challenges suggest that further collaboration between administrators, practitioners, and researchers is essential. Despite the competing Primary Health Care Research \& Development 2018; 19: 96-98 
demands, each of these professional groups could greatly benefit from a more comprehensive understanding of the role of mental health and physical health comorbidities. One way to develop evidence-based treatments is to appropriately understand physical and mental health comorbidities and the role of mental health conditions in outcomes of care.

\section{Conclusion}

Researchers often rely on comorbidity indices to statistically control for severity using morbidity and mortality measures, including the Charlson Comorbidity Index and the Elixhauser Comorbidity Measure. However, such measures account for physical health conditions and entirely overlook mental health conditions, which are known to be associated with patient morbidity and mortality. By not including mental health conditions in comorbidity indices, analyses are left open to potential confounders in the form of unaccounted for mental health conditions, and prognostic use may be underestimating morbidity and mortality. A new measure should include mental health and substance use, and will need to be re-validated to ensure its reliability.

To further the field of health-related research, an index for assessing comorbidity of mental health and substance abuse is essential and long overdue. Even better, a new measure should take into account both physical and behavioral health issues together to aid researchers in the transformation from siloed physical and mental health to a model where these two fields are no longer artificially separated between the mind and body.

\section{Financial Support}

This research received no specific grant from any funding agency, commercial or not-for-profit sectors.

\section{Conflicts of Interest}

None.

\section{References}

Boardman, J.B. 2006: Health access and integration for adults with serious and persistent mental illness. Families, Systems, \& Health 24, 3-18.

Primary Health Care Research \& Development 2018; 19: 96-98
Charlson, M.E., Pompei, P., Ales, K.L. and Mackenzie, C.R. 1987: A new method of classifying prognostic comorbidity in longitudinal studies: development and validation. Journal of Chronic Diseases 40, 373-83.

D'Hoore, W., Bouckaert, A. and Tilquin, C. 1996: Practical considerations on the use of the Charlson comorbidity index with administrative data bases. Journal of Clinical Epidemiology 49, 1429-433.

Dickey, B., Normand, S.L., Weiss, R.D., Drake, R.E. and Azeni, H. 2002: Medical morbidity, mental illness, and substance use disorders. Psychiatric Service 53, 861-67.

Druss, B.G. and Walker, E.R. 2011: Mental disorders and medical comorbidity. Synthesis Project: Research Synthesis Report 21, 1-26.

Elixhauser, A., Steiner, C., Harris, D.R. and Coffey, R.M. 1998: Comorbidity measures for use with administrative data. Medical Care 36, 8-27.

Jones, D.R., Macias, C., Barreira, P.J., Fisher, W.H., Hargreaves, W.A. and Harding, C.M. 2004: Prevalence, severity, and co-occurrence of chronic physical health problems of persons with serious mental illness. Psychiatric Service 55, 1250-257.

Kessler, R.C., Berglund, P., Demler, O., Jin, R., Merikangas, K.R. and Walters, E.E. 2005a: Lifetime prevalence and age-of-onset distributions of DSM-IV disorders in the National Comorbidity Survey Replication. Archives of General Psychiatry 62, 593-602.

Kessler, R.C., Chiu, W.T., Demler, O., Merikangas, K.R. and Walters, E.E. 2005b: Prevalence, severity, and comorbidity of 12-month DSM-IV disorders in the National Comorbidity Survey Replication. Archives of General Psychiatry 62, 617-627.

Klabunde, C.N., Potosky, A.L., Legler, JM. and Warren, J.L. 2000: Development of a comorbidity index using physician claims data. Journal of Clinical Epidemiology 53, 1258-267.

Mehta, N. 2011: Mind-body Dualism: a critique from a health perspective. Mens Sana Monographs 9, 202-9.

Newman, S.C. and Bland, R.C. 1991: Mortality in a cohort of patients with schizophrenia: a record linkage study. Canadian Journal of Psychiatry 36, 239-45.

Regier, D.A., Farmer, M.E., Rae, D.S., Locke, B.Z., Keith, S. J., Judd, L.L. and Goodwin, F.K. 1990: Comorbidity of mental disorders with alcohol and other drug abuse. Results from the Epidemiologic Catchment Area (ECA) Study. JAMA 264, 2511-518.

SAMHSA. 2014. Results from the 2013 National Survey on drug use and health: mental health findings. NSDUH series H-49 Rockville, MD: Substance Abuse and Mental Health Services Administration.

Sokal, J., Messias, E., Dickerson, F.B., Kreyenbuhl, J., Brown, C.H., Goldberg, R.W. and Dixon, L.B. 2004: Comorbidity of medical illnesses among adults with serious mental illness who are receiving community psychiatric services. Journal of Nervous and Mental Disease 192, 421-427. 